\title{
Conditional convergence for randomly weighted sums of random variables based on conditional residual $h$-integrability
}

\author{
Aiting Shen ${ }^{*}$, Ranchao Wu, Yan Chen and Yu Zhou
}

\section{"Correspondence:}

Empress201010@126.com School of Mathematical Science,

Anhui University, Hefei, 230601, P.R. China

\section{Springer}

\begin{abstract}
Let $\left\{X_{n k}, u_{n} \leq k \leq v_{n}, n \geq 1\right\}$ and $\left\{A_{n k}, u_{n} \leq k \leq v_{n}, n \geq 1\right\}$ be two arrays of random variables defined on the same probability space $(\Omega, \mathcal{A}, P)$ and $\mathcal{B}_{n}$ be sub- $\sigma$-algebras of $\mathcal{A}$. Let $r>0$ be a constant. In this paper, we introduce some concepts of conditional residual $h$-integrability such as conditionally residually $h$-integrable relative to $\mathcal{B}_{n}$ concerning the array $\left\{A_{n k}\right\}$ with exponent $r$ and conditionally strongly residually $h$-integrable relative to $\mathcal{B}_{n}$ concerning the array $\left\{A_{n k}\right\}$ with exponent $r$. These concepts are more general than some known setting of randomly weighted sums of random variables. Based on the conditions of conditional residual $h$-integrability with exponent $r$ and conditional strongly residual $h$-integrability with exponent $r$, we obtain the conditional mean convergence and conditional almost sure convergence for randomly weighted sums.
\end{abstract}

MSC: 60F15; 60F25

Keywords: uniform integrability; randomly weighted sums; conditional mean convergence; conditional almost sure convergence; conditionally residually integrable; conditionally strongly residually integrable

\section{Introduction}

Throughout this paper, all the random variables are defined on the same probability space $(\Omega, \mathcal{A}, P)$, and we let $\mathcal{B}$ and $\mathcal{B}_{n}$ be sub- $\sigma$-algebras of $\mathcal{A}$. Suppose that $\left\{u_{n}, n \geq 1\right\}$ and $\left\{v_{n}, n \geq\right.$ $1\}$ are two sequences of integers (not necessary positive or finite) such that $u_{n}<v_{n}$ for all $n \geq 1$ and $v_{n}-u_{n} \rightarrow \infty$ as $n \rightarrow \infty$. Let $\left\{k_{n}, n \geq 1\right\}$ be a sequence of positive numbers such that $k_{n} \rightarrow \infty$ as $n \rightarrow \infty$. Let $I(A)$ be the indicator function of the set $A$ and $P^{\mathcal{B}}(A)=E^{\mathcal{B}}\left(I_{A}\right)$. Moreover, let $\{h(n), n \geq 1\}$ be an increasing sequence of positive constants with $h(n) \uparrow \infty$ as $n \uparrow \infty$.

\subsection{Brief review}

Conditional limit theorems play a key role in the study of statistical inference. A typical example of statistical application of conditional limit theorems is in the study of statistical inference for some branching processes such as the Galton-Watson process (see, e.g., Basawa and Prakasa Rao [1]). Let $\left\{Z_{0}=1, Z_{n}, n \geq 1\right\}$ be a Galton-Watson process with mean offspring $\Theta$. This process can be studied by means of the following autoregressive type

(C) 2013 Shen et al: licensee Springer. This is an Open Access article distributed under the terms of the Creative Commons Attribution License (http://creativecommons.org/licenses/by/2.0), which permits unrestricted use, distribution, and reproduction in any medium, provided the original work is properly cited. 
model:

$$
Z_{n+1}=\Theta Z_{n}+Z_{n}^{1 / 2} U_{n+1}, \quad n \geq 0
$$

where $\left\{U_{k}, k \geq 0\right\}$ is the sequence of error random variables.

To estimate the mean offspring $\Theta$ from a realization $\left\{Z_{0}=1, Z_{n}, n \geq 1\right\}$, the maximum likelihood estimator of $\Theta$ is $\hat{\Theta}_{n}=\left(\sum_{k=1}^{n} Z_{k-1}\right)^{-1}\left(\sum_{k=1}^{n} Z_{k}\right)$, which coincides with the 'leastsquares' estimator of $\Theta$ obtained by minimizing $\sum_{k=0}^{n} U_{k}^{2}$ with respect to $\Theta$.

The study of asymptotic properties of $\hat{\Theta}_{n}$ leads to a conditional limit theorem since, as it is detailed in Basawa and Prakasa Rao [1], these asymptotic properties of $\hat{\Theta}_{n}$ depend on the event of non-extinction of the process. For more details about the conditional limit theorem, one can refer to Roussas [2], Leek [3], Ordóñez Cabrera et al. [4], and so forth.

The main purpose of the paper is to introduce some new concepts of conditional residual $h$-integrability. Based on these conditions, we study the conditional mean convergence and conditional almost sure convergence for randomly weighted sums of random variables.

\subsection{Some concepts of integrability}

It is well known that the notion of uniform integrability plays the central role in establishing weak laws of large numbers. In this section, we recall some concepts of integrability.

Firstly, we recall the notion of conditional covariance. The interested reader can find further results in Chow and Teicher [5] and Roussas [2]. Let $X$ and $Y$ be random variables defined on a probability space $(\Omega, \mathcal{A}, P)$ with $E X^{2}<\infty$ and $E Y^{2}<\infty$. Prakasa Rao [6] defined the notion of conditional covariance of $X$ and $Y$ given $\mathcal{B}$ (B- -covariance for short) as

$$
\operatorname{Cov}^{\mathcal{B}}(X, Y)=E^{\mathcal{B}}\left(\left(X-E^{\mathcal{B}} X\right)\left(Y-E^{\mathcal{B}} Y\right)\right)
$$

where $E^{\mathcal{B}} Z$ denotes the conditional expectation of a random variable $Z$ given $\mathcal{B}$. In contrast to the ordinary concept of variance, conditional variance of $X$ given $\mathcal{B}$ is defined as $\operatorname{Var}^{\mathcal{B}} X=\operatorname{Cov}^{\mathcal{B}}(X, X)$.

In the following, we present some concepts of uniform integrability.

The classical notion of uniform integrability of a sequence of integrable random variables $\left\{X_{n}, n \geq 1\right\}$ is defined through the condition

$$
\lim _{a \rightarrow \infty} \sup _{n \geq 1} E\left|X_{n}\right| I\left(\left|X_{n}\right|>a\right)=0 .
$$

Landers and Rogge [7] proved that the uniform integrability condition is sufficient in order that a sequence of pairwise independent random variables verifies the weak laws of large numbers.

Chandra [8] introduced the notion of Cesàro uniform integrability which is weaker than uniform integrability. A sequence of integrable random variables $\left\{X_{n}, n \geq 1\right\}$ is said to be Cesàro uniformly integrable if

$$
\lim _{a \rightarrow \infty} \sup _{n \geq 1} \frac{1}{m_{n}} \sum_{k=1}^{m_{n}} E\left|X_{k}\right| I\left(\left|X_{k}\right|>a\right)=0,
$$

where $\left\{m_{n}, n \geq 1\right\}$ is a sequence of positive integers such that $m_{n} \rightarrow \infty$ as $n \rightarrow \infty$. 
Ordóñez Cabrera [9], by studying the weak convergence for weighted sums of random variables, introduced the condition of uniform integrability concerning the weights, which is weaker than uniform integrability and includes the Cesàro uniform integrability as a special case.

Definition 1.1 Let $\left\{X_{n k}, u_{n} \leq k \leq v_{n}, n \geq 1\right\}$ be an array of random variables, and let $\left\{a_{n k}, u_{n} \leq k \leq v_{n}, n \geq 1\right\}$ be an array of constants with $\sum_{k=u_{n}}^{v_{n}}\left|a_{n k}\right| \leq C$ for all $n \geq 1$ and some constant $C>0$. The array $\left\{X_{n k}\right\}$ is $\left\{a_{n k}\right\}$-uniformly integrable if

$$
\lim _{a \rightarrow \infty} \sup _{n \geq 1} \sum_{k=u_{n}}^{v_{n}}\left|a_{n k}\right| E\left|X_{n k}\right| I\left(\left|X_{n k}\right|>a\right)=0 .
$$

Sung [10] gave a slight generalization and introduced the concept of Cesàro-type uniform integrability with exponent $r$ as follows.

Definition 1.2 Let $\left\{X_{n k}, u_{n} \leq k \leq v_{n}, n \geq 1\right\}$ be an array of random variables and $r>0$. The array $\left\{X_{n k}\right\}$ is said to be Cesàro-type uniformly integral with exponent $r$ if

$$
\sup _{n \geq 1} \frac{1}{k_{n}} \sum_{k=u_{n}}^{v_{n}} E\left|X_{n k}\right|^{r}<\infty
$$

and

$$
\lim _{a \rightarrow \infty} \sup _{n \geq 1} \frac{1}{k_{n}} \sum_{k=u_{n}}^{v_{n}} E\left|X_{n k}\right|^{r} I\left(\left|X_{n k}\right|^{r}>a\right)=0 .
$$

Based on the Cesàro-type uniform integrability with exponent $r$ for some $0<r<2$, Sung [10] obtained the weak law of large numbers for the array $\left\{X_{n k}, u_{n} \leq k \leq v_{n}, n \geq 1\right\}$.

Chandra and Goswami [11] introduced the concept of Cesàro $\alpha$-integrability $(\alpha>0)$ and showed that Cesàro $\alpha$-integrability for any $\alpha>0$ is weaker than Cesàro uniform integrability. Under the condition of Cesàro $\alpha$-integrability for some $\alpha>1 / 2$, they obtained the weak law of large numbers for a sequence of pairwise independent random variables. They also proved that Cesàro $\alpha$-integrability for appropriate $\alpha$ is also sufficient for the weak law of large numbers to hold for certain special dependent sequences of random variables.

Inspired by the concept of Cesàro $\alpha$-integrability, Chandra and Goswami [12] introduced the following concept of residual Cesàro $(\alpha, r)$-integrability, which is weaker than Cesàro $\alpha$-integrability.

Definition 1.3 Let $\alpha \in(0, \infty), r \in(0, \infty)$. A sequence of random variables $\left\{X_{n}, n \geq 1\right\}$ is said to be residually Cesàro $(\alpha, r)$-integrable ( $\mathrm{RCI}(\alpha, r)$ for short) if

$$
\sup _{n \geq 1} \frac{1}{n} \sum_{k=1}^{n} E\left|X_{k}\right|^{r}<\infty
$$

and

$$
\lim _{n \rightarrow \infty} \frac{1}{n} \sum_{k=1}^{n} E\left(\left|X_{k}\right|-k^{\alpha}\right)^{r} I\left(\left|X_{k}\right|>k^{\alpha}\right)=0 .
$$


It is easily seen that RCI $(\alpha, r)$ integrability with $r=1$ is $\mathrm{RCI}(\alpha)$ integrability ( $c f$. Chandra and Goswami [12]).

Ordóñez Cabrera and Volodin [13] introduced the notion of $h$-integrability for an array of random variables concerning an array of constants $\left\{a_{n k}\right\}$ and showed that this concept is weaker than Cesàro uniform integrability, $\left\{a_{n k}\right\}$-uniform integrability and Cesàro $\alpha$ integrability. The notion of $h$-integrability for an array of random variables concerning an array of constants $\left\{a_{n k}\right\}$ is as follows.

Definition 1.4 Let $\left\{X_{n k}, u_{n} \leq k \leq v_{n}, n \geq 1\right\}$ be an array of random variables, and let $\left\{a_{n k}, u_{n} \leq k \leq v_{n}, n \geq 1\right\}$ be an array of constants with $\sum_{k=u_{n}}^{v_{n}}\left|a_{n k}\right| \leq C$ for all $n \geq 1$ and some constant $C>0$. The array $\left\{X_{n k}\right\}$ is said to be $h$-integrable concerning the array of constants $\left\{a_{n k}\right\}$ if

$$
\sup _{n \geq 1} \sum_{k=u_{n}}^{v_{n}}\left|a_{n k}\right| E\left|X_{n k}\right|<\infty
$$

and

$$
\lim _{n \rightarrow \infty} \sum_{k=u_{n}}^{v_{n}}\left|a_{n k}\right| E\left|X_{n k}\right| I\left(\left|X_{n k}\right|>h(n)\right)=0 .
$$

Sung et al. [14] generalized the notion of $h$-integrability and introduced the concept of $h$-integrability with exponent $r$. They also proved that the concept of $h$-integrability with exponent $r$ is strictly weaker than the concept of Cesàro-type uniformly integral with exponent $r$. The concept of $h$-integrability with exponent $r$ is as follows.

Definition 1.5 Let $\left\{X_{n k}, u_{n} \leq k \leq v_{n}, n \geq 1\right\}$ be an array of random variables and $r>0$. The array $\left\{X_{n k}, u_{n} \leq k \leq v_{n}, n \geq 1\right\}$ is said to be $h$-integrable with exponent $r$ if

$$
\sup _{n \geq 1} \frac{1}{k_{n}} \sum_{k=u_{n}}^{v_{n}} E\left|X_{n k}\right|^{r}<\infty
$$

and

$$
\lim _{n \rightarrow \infty} \frac{1}{k_{n}} \sum_{k=u_{n}}^{v_{n}} E\left|X_{n k}\right|^{r} I\left(\left|X_{n k}\right|^{r}>h(n)\right)=0 .
$$

At the same time, Yuan and Tao [15] introduced $R$ - $h$-integrability concerning the array of constants $\left\{a_{n k}\right\}$ as follows.

Definition 1.6 Let $\left\{X_{n k}, u_{n} \leq k \leq v_{n}, n \geq 1\right\}$ be an array of random variables, and let $\left\{a_{n k}, u_{n} \leq k \leq v_{n}, n \geq 1\right\}$ be an array of constants. The array $\left\{X_{n k}, u_{n} \leq k \leq v_{n}, n \geq 1\right\}$ is said to be residually h-integrable ( $R$-h-integrable for short) concerning the array of constants $\left\{a_{n k}\right\}$ if

$$
\sup _{n \geq 1} \sum_{k=u_{n}}^{v_{n}}\left|a_{n k}\right| E\left|X_{n k}\right|<\infty
$$


and

$$
\lim _{n \rightarrow \infty} \sum_{k=u_{n}}^{v_{n}}\left|a_{n k}\right| E\left(\left|X_{n k}\right|-h(n)\right) I\left(\left|X_{n k}\right|>h(n)\right)=0 .
$$

Inspired by the concepts above, recently, Ordóñez Cabrera et al. [4] introduced the notion of conditional residual $h$-integrability relative to the sequence $\left\{\mathcal{B}_{n}\right\}$ as follows.

Definition 1.7 Let $\left\{X_{n k}, u_{n} \leq k \leq v_{n}, n \geq 1\right\}$ and $\left\{A_{n k}, u_{n} \leq k \leq v_{n}, n \geq 1\right\}$ be two arrays of random variables. The array $\left\{X_{n k}\right\}$ is said to be conditionally residually $h$-integrable relative to $\mathcal{B}_{n}\left(\mathcal{B}_{n}-C R\right.$ - $h$-integrable for short) concerning the array $\left\{A_{n k}\right\}$ if

$$
\sup _{n \geq 1} \sum_{k=u_{n}}^{v_{n}}\left|A_{n k}\right| E^{\mathcal{B}_{n}}\left|X_{n k}\right|^{r}<\infty \quad \text { a.s. }
$$

and

$$
\lim _{n \rightarrow \infty} \sum_{k=u_{n}}^{v_{n}}\left|A_{n k}\right| E^{\mathcal{B}_{n}}\left(\left|X_{n k}\right|-h(n)\right) I\left(\left|X_{n k}\right|>h(n)\right)=0 \quad \text { a.s. }
$$

It is easily seen that if $A_{n k} \equiv a_{n k}$ are constants and $\mathcal{B}_{n}=\{\emptyset, \Omega\}$ for all $n \geq 1$, then the concept of $\mathcal{B}_{n}-C R$ - $h$-integrability concerning the array $\left\{A_{n k}\right\}$ reduces to the concept of $R$ - $h$-integrability concerning the array of constants $\left\{a_{n k}\right\}$.

To obtain a conditional strong convergence result, Ordóñez Cabrera et al. [4] introduced the following concept of conditional strongly residual $h$-integrability relative to the sequence $\mathcal{B}_{n}$, which is stronger than that of $\mathcal{B}_{n}-C R-h$-integrability concerning the array $\left\{A_{n k}\right\}$.

Definition 1.8 Let $\left\{X_{n k}, u_{n} \leq k \leq v_{n}, n \geq 1\right\}$ and $\left\{A_{n k}, u_{n} \leq k \leq v_{n}, n \geq 1\right\}$ be two arrays of random variables. The array $\left\{X_{n k}\right\}$ is said to be conditionally strongly residually $h$ integrable relative to $\mathcal{B}_{n}\left(\mathcal{B}_{n}\right.$-CSR- $h$-integrable for short) concerning the array $\left\{A_{n k}\right\}$ if

$$
\sup _{n \geq 1} \sum_{k=u_{n}}^{v_{n}}\left|A_{n k}\right| E^{\mathcal{B}_{n}}\left|X_{n k}\right|<\infty \quad \text { a.s. }
$$

and

$$
\sum_{n=1}^{\infty} \sum_{k=u_{n}}^{v_{n}}\left|A_{n k}\right| E^{\mathcal{B}_{n}}\left(\left|X_{n k}\right|-h(n)\right) I\left(\left|X_{n k}\right|>h(n)\right)<\infty \quad \text { a.s. }
$$

Inspired by the concepts above, we introduce some new concepts of conditional residual $h$-integrability such as conditionally residually $h$-integrable relative to $\mathcal{B}_{n}$ concerning the array $\left\{A_{n k}\right\}$ with exponent $r$ and conditionally strongly residually $h$-integrable relative to $\mathcal{B}_{n}$ concerning the array $\left\{A_{n k}\right\}$ with exponent $r$. These concepts are more general than some known setting of randomly weighted sums of random variables. Based on the conditions of conditional residual $h$-integrability with exponent $r$ and conditional strongly residual $h$-integrability with exponent $r$, we study the conditional mean convergence and conditional almost sure convergence for randomly weighted sums. 


\section{Conditional mean convergence for randomly weighted sums}

In this section, we study the conditional mean convergence for randomly weighted sums. Inspired by the concepts stated in Section 1, we introduce the notion of conditional residual $h$-integrability relative to the sequence $\left\{\mathcal{B}_{n}\right\}$ with exponent $r$ as follows.

Definition 2.1 Let $\left\{X_{n k}, u_{n} \leq k \leq v_{n}, n \geq 1\right\}$ and $\left\{A_{n k}, u_{n} \leq k \leq v_{n}, n \geq 1\right\}$ be two arrays of random variables. Let $r>0$ be a constant. The array $\left\{X_{n k}\right\}$ is said to be conditionally residually $h$-integrable relative to $\mathcal{B}_{n}\left(\mathcal{B}_{n}\right.$-CR-h-integrable for short) concerning the array $\left\{A_{n k}\right\}$ with exponent $r$ if

$$
\sup _{n \geq 1} \sum_{k=u_{n}}^{v_{n}}\left|A_{n k}\right| E^{\mathcal{B}_{n}}\left|X_{n k}\right|^{r}<\infty \quad \text { a.s. }
$$

and

$$
\lim _{n \rightarrow \infty} \sum_{k=u_{n}}^{v_{n}}\left|A_{n k}\right| E^{\mathcal{B}_{n}}\left(\left|X_{n k}\right|-h^{1 / r}(n)\right)^{r} I\left(\left|X_{n k}\right|^{r}>h(n)\right)=0 \quad \text { a.s. }
$$

Remark 2.1 In Definition 2.1, if the exponent $r$ takes value 1, then we can get the concept of conditionally residually $h$-integrable relative to $\mathcal{B}_{n}\left(\mathcal{B}_{n}\right.$-CR-h-integrable for short) concerning the array $\left\{A_{n k}\right\}$, which was introduced by Ordóñez Cabrera et al. [4]. Just as Ordóñez Cabrera et al. [4] stated that the concept of $\mathcal{B}_{n}-C R$ - $h$-integrable concerning the array $\left\{A_{n k}\right\}$ is a conditional extension to the more general setting of randomly weighted sums of random variables of (i) the concept of residual Cesàro $\alpha$-integrability introduced by Chandra and Goswami [12] and (ii) the concept of residual $h$-integrability concerning an array of constants introduced by Yuan and Tao [15], the concept of $\mathcal{B}_{n}$-CR-h-integrable concerning the array $\left\{A_{n k}\right\}$ with exponent $r$ is more general.

Remark 2.2 If $A_{n k} \equiv a_{n k}$ are constants, and $\mathcal{B}_{n}=\{\emptyset, \Omega\}$ for all $n \geq 1$, then the preceding definition reduces to the following new concept of residual $h$-integrability concerning the array of constants $\left\{a_{n k}\right\}$ with exponent $r$.

Definition 2.2 Let $\left\{X_{n k}, u_{n} \leq k \leq v_{n}, n \geq 1\right\}$ be an array of random variables, and let $\left\{a_{n k}, u_{n} \leq k \leq v_{n}, n \geq 1\right\}$ be an array of constants. Let $r>0$ be a constant. The array $\left\{X_{n k}\right\}$ is said to be residually $h$-integrable ( $R$ - $h$-integrable for short) concerning the array $\left\{a_{n k}\right\}$ with exponent $r$ if

$$
\sup _{n \geq 1} \sum_{k=u_{n}}^{v_{n}}\left|a_{n k}\right| E\left|X_{n k}\right|^{r}<\infty
$$

and

$$
\lim _{n \rightarrow \infty} \sum_{k=u_{n}}^{v_{n}}\left|a_{n k}\right| E\left(\left|X_{n k}\right|-h^{1 / r}(n)\right)^{r} I\left(\left|X_{n k}\right|^{r}>h(n)\right)=0 .
$$

Remark 2.3 Let $\left\{h_{1}(n), n \geq 1\right\}$ and $\left\{h_{2}(n), n \geq 1\right\}$ be two positive monotonically increasing to infinity sequences such that $h_{2}(n) \geq h_{1}(n)$ for all sufficiently large $n$. Then $\mathcal{B}_{n}$ - $C R-h_{1}$ integrability with exponent $r$ implies $\mathcal{B}_{n}$-CR-h $h_{2}$-integrability with exponent $r$. 
Remark 2.4 Take $a_{n k}=\frac{1}{k_{n}}$ for each $k$ in Definition 2.2, where $\left\{k_{n}\right\}$ is a sequence of positive numbers such that $k_{n} \rightarrow \infty$ as $n \rightarrow \infty$. Note that

$$
\left(\left|X_{n k}\right|-h^{1 / r}(n)\right)^{r} I\left(\left|X_{n k}\right|^{r}>h(n)\right) \leq\left|X_{n k}\right|^{r} I\left(\left|X_{n k}\right|^{r}>h(n)\right)
$$

so the concept of $R$ - $h$-integrable concerning the array $\left\{a_{n k}\right\}$ with exponent $r$ is much weaker than the notion of $h$-integrability with exponent $r$, which was introduced by Sung [14].

Based on the condition of $\mathcal{B}_{n}$-CR-h-integrability concerning the array $\left\{A_{n k}\right\}$ with exponent $r$, we study the conditional mean convergence for randomly weighted sums. Our main result is as follows.

Theorem 2.1 Let $\left\{X_{n k}, u_{n} \leq k \leq v_{n}, n \geq 1\right\}$ be an array of random variables, and let $\left\{A_{n k}, u_{n} \leq k \leq v_{n}, n \geq 1\right\}$ be an array of nonnegative random variables such that for each $n \geq 1,\left\{A_{n k}, u_{n} \leq k \leq v_{n}\right\}$ are $\mathcal{B}_{n}$-measurable. Suppose that

(i) $\left\{X_{n k}\right\}$ is $\mathcal{B}_{n}$-CR-h-integrable concerning the array $\left\{A_{n k}\right\}$ with exponent $0<r<1$;

(ii) $h(n) \sup _{u_{n} \leq k \leq v_{n}} A_{n k} \rightarrow 0$ a.s. as $n \rightarrow \infty$.

Let $S_{n}=\sum_{k=u_{n}}^{v_{n}} A_{n k}^{1 / r} X_{n k}, n \geq 1$. Then $E^{\mathcal{B}_{n}}\left|S_{n}\right|^{r} \rightarrow 0$ a.s. as $n \rightarrow \infty$.

Proof For each $n \geq 1$ and $u_{n} \leq k \leq v_{n}$, we define, by using the method of continuous truncation, the following:

$$
Y_{n k}=-h^{1 / r}(n) I\left(X_{n k}<-h^{1 / r}(n)\right)+X_{n k} I\left(\left|X_{n k}\right| \leq h^{1 / r}(n)\right)+h^{1 / r}(n) I\left(X_{n k}>h^{1 / r}(n)\right)
$$

and $Z_{n k}=X_{n k}-Y_{n k}$. Hence, we can write that

$$
S_{n}=\sum_{k=u_{n}}^{v_{n}} A_{n k}^{1 / r} X_{n k}=\sum_{k=u_{n}}^{v_{n}} A_{n k}^{1 / r} Y_{n k}+\sum_{k=u_{n}}^{v_{n}} A_{n k}^{1 / r} Z_{n k} \doteq S_{1 n}+S_{2 n}, \quad n \geq 1,
$$

and we estimate the conditional expectation of each of these terms separately. With regard to $S_{2 n}$, since $\left|Z_{n k}\right|=\left(\left|X_{n k}\right|-h^{1 / r}(n)\right) I\left(\left|X_{n k}\right|^{r}>h(n)\right)$, we have by $C_{r}$ 's inequality and Definition 1.8 that

$$
\begin{aligned}
E^{\mathcal{B}_{n}}\left|S_{2 n}\right|^{r} & =E^{\mathcal{B}_{n}}\left|\sum_{k=u_{n}}^{v_{n}} A_{n k}^{1 / r} Z_{n k}\right|^{r} \\
& \leq \sum_{k=u_{n}}^{v_{n}} A_{n k} E^{\mathcal{B}_{n}}\left|Z_{n k}\right|^{r} \\
& =\sum_{k=u_{n}}^{v_{n}} A_{n k} E^{\mathcal{B}_{n}}\left(\left|X_{n k}\right|-h^{1 / r}(n)\right)^{r} I\left(\left|X_{n k}\right|^{r}>h(n)\right) \rightarrow 0 \quad \text { a.s. as } n \rightarrow \infty .
\end{aligned}
$$

For $S_{1 n}$, we initially prove $E^{\mathcal{B}_{n}}\left|S_{1 n}\right| \rightarrow 0$ a.s. as $n \rightarrow \infty$. Noting that

$$
\left|Y_{n k}\right|=\min \left\{\left|X_{n k}\right|, h^{1 / r}(n)\right\}, \quad u_{n} \leq k \leq v_{n}, n \geq 1,
$$


we have by (2.1), condition (ii) and Definition 1.8 that

$$
\begin{aligned}
E^{\mathcal{B}_{n}}\left|S_{1 n}\right| & \leq \sum_{k=u_{n}}^{v_{n}} A_{n k}^{1 / r} E^{\mathcal{B}_{n}}\left(\left|Y_{n k}\right|^{r} \cdot\left|Y_{n k}\right|^{1-r}\right) \leq h^{(1-r) / r}(n) \sum_{k=u_{n}}^{v_{n}} A_{n k}^{1 / r} E^{\mathcal{B}_{n}}\left|X_{n k}\right|^{r} \\
& \leq\left(h(n) \sup _{u_{n} \leq k \leq v_{n}} A_{n k}\right)^{\frac{1-r}{r}} \sum_{k=u_{n}}^{v_{n}} A_{n k} E^{\mathcal{B}_{n}}\left|X_{n k}\right|^{r} \rightarrow 0 \quad \text { a.s. as } n \rightarrow \infty,
\end{aligned}
$$

which implies that

$$
E^{\mathcal{B}_{n}}\left|S_{1 n}\right| \rightarrow 0 \quad \text { a.s. as } n \rightarrow \infty .
$$

By Jensen's inequality for conditional expectations (see, e.g., Chow and Teicher [5], p. 217), we have by (2.2) that

$$
E^{\mathcal{B}_{n}}\left|S_{1 n}\right|^{r}=\left[\left(E^{\mathcal{B}_{n}}\left|S_{1 n}\right|^{r}\right)^{1 / r}\right]^{r} \leq\left(E^{\mathcal{B}_{n}}\left|S_{1 n}\right|\right)^{r} \rightarrow 0 \quad \text { a.s. as } n \rightarrow \infty .
$$

Therefore, we have by $C_{r}$ 's inequality that

$$
E^{\mathcal{B}_{n}}\left|S_{n}\right|^{r} \leq E^{\mathcal{B}_{n}}\left|S_{1 n}\right|^{r}+E^{\mathcal{B}_{n}}\left|S_{2 n}\right|^{r} \rightarrow 0 \quad \text { a.s. as } n \rightarrow \infty .
$$

This completes the proof of the theorem.

\section{Conditional almost sure convergence for randomly weighted sums}

To obtain a conditional strong convergence result, we introduce the concept of conditional strongly residual $h$-integrability relative to the sequence $\mathcal{B}_{n}$ with exponent $r$ as follows.

Definition 3.1 Let $\left\{X_{n k}, u_{n} \leq k \leq v_{n}, n \geq 1\right\}$ and $\left\{A_{n k}, u_{n} \leq k \leq v_{n}, n \geq 1\right\}$ be two arrays of random variables. Let $r>0$ be a constant. The array $\left\{X_{n k}\right\}$ is said to be conditionally strongly residually $h$-integrable relative to $\mathcal{B}_{n}\left(\mathcal{B}_{n}\right.$-CSR-h-integrable for short) concerning the array $\left\{A_{n k}\right\}$ with exponent $r$ if

$$
\sup _{n \geq 1} \sum_{k=u_{n}}^{v_{n}}\left|A_{n k}\right| E^{\mathcal{B}_{n}}\left|X_{n k}\right|^{r}<\infty \quad \text { a.s. }
$$

and

$$
\sum_{n=1}^{\infty} \sum_{k=u_{n}}^{v_{n}}\left|A_{n k}\right| E^{\mathcal{B}_{n}}\left(\left|X_{n k}\right|-h^{1 / r}(n)\right)^{r} I\left(\left|X_{n k}\right|^{r}>h(n)\right)<\infty \quad \text { a.s. }
$$

Remark 3.1 In Definition 3.1, if the exponent $r$ takes value 1, then we can get the conditionally strongly residually $h$-integrable relative to $\mathcal{B}_{n}\left(\mathcal{B}_{n}\right.$-CSR- $h$-integrable, for short) concerning the array $\left\{A_{n k}\right\}$, which was introduced by Ordóñez Cabrera et al. [4].

Remark 3.2 If $A_{n k} \equiv a_{n k}$ are constants, and $\mathcal{B}_{n}=\{\emptyset, \Omega\}$ for all $n \geq 1$, then the preceding definition reduces to the following new concept of strongly residual $h$-integrability concerning the array of constants $\left\{a_{n k}\right\}$ with exponent $r$. 
Definition 3.2 Let $\left\{X_{n k}, u_{n} \leq k \leq v_{n}, n \geq 1\right\}$ be an array of random variables, and let $\left\{a_{n k}, u_{n} \leq k \leq v_{n}, n \geq 1\right\}$ be an array of constants. Let $r>0$ be a constant. The array $\left\{X_{n k}\right\}$ is said to be strongly residually $h$-integrable ( $S R$ - $h$-integrable for short) concerning the array $\left\{a_{n k}\right\}$ with exponent $r$ if

$$
\sup _{n \geq 1} \sum_{k=u_{n}}^{v_{n}}\left|a_{n k}\right| E\left|X_{n k}\right|^{r}<\infty
$$

and

$$
\sum_{n=1}^{\infty} \sum_{k=u_{n}}^{v_{n}}\left|a_{n k}\right| E\left(\left|X_{n k}\right|-h^{1 / r}(n)\right)^{r} I\left(\left|X_{n k}\right|^{r}>h(n)\right)<\infty
$$

Remark 3.3 It is easily seen that the concept of $\mathcal{B}_{n}$-CSR-h-integrability with exponent $r$ is stronger than the concept of $\mathcal{B}_{n}$-CR-h-integrability with exponent $r$. Likewise, the unconditional concept of $S R$-h-integrability with exponent $r$ is stronger than the concept of $R$ - $h$-integrability with exponent $r$.

We now establish a strong version of Theorem 2.1 under the condition of $\mathcal{B}-C S R$ - $h$ integrability (i.e., when $\mathcal{B}_{n}=\mathcal{B}$, a sub- $\sigma$-algebra of $\mathcal{A}$, for all $n \geq 1$ ).

Theorem 3.1 Let $\left\{X_{n k}, u_{n} \leq k \leq v_{n}, n \geq 1\right\}$ be an array of random variables, and let $\left\{A_{n k}, u_{n} \leq k \leq v_{n}, n \geq 1\right\}$ be an array of nonnegative $\mathcal{B}$-measurable random variables. Suppose that

(i) $\left\{X_{n k}\right\}$ is $\mathcal{B}$-CSR-h-integrable concerning the array $\left\{A_{n k}\right\}$ with exponent $0<r<1$;

(ii) $\sum_{n=1}^{\infty}\left(h(n) \sup _{u_{n} \leq k \leq v_{n}} A_{n k}\right)^{\frac{1-r}{r}}<\infty$ a.s.

Then $S_{n}=\sum_{k=u_{n}}^{v_{n}} A_{n k}^{1 / r} X_{n k} \rightarrow 0$ a.s. as $n \rightarrow \infty$.

Proof We use the same notations as those in Theorem 2.1 and set $\mathcal{B}_{n}=\mathcal{B}$ for each $n \geq 1$. Then $S_{n}=S_{1 n}+S_{2 n}$ for each $n \geq 1$, and we estimate each of these terms separately.

Condition (i) implies via the nonnegativity of every summand that

$$
E^{\mathcal{B}_{n}}\left[\sum_{n=1}^{\infty} \sum_{k=u_{n}}^{v_{n}} A_{n k}\left(\left|X_{n k}\right|-h^{1 / r}(n)\right)^{r} I\left(\left|X_{n k}\right|^{r}>h(n)\right)\right]<\infty \quad \text { a.s. }
$$

which implies that

$$
\sum_{n=1}^{\infty} \sum_{k=u_{n}}^{v_{n}} A_{n k}\left(\left|X_{n k}\right|-h^{1 / r}(n)\right)^{r} I\left(\left|X_{n k}\right|^{r}>h(n)\right)<\infty \quad \text { a.s. }
$$

Note that $\left|Z_{n k}\right|=\left(\left|X_{n k}\right|-h^{1 / r}(n)\right) I\left(\left|X_{n k}\right|^{r}>h(n)\right)$, we have by (3.1) that

$$
\begin{aligned}
\left|S_{2 n}\right|^{r} & =\left|\sum_{k=u_{n}}^{v_{n}} A_{n k}^{1 / r} Z_{n k}\right|^{r} \leq \sum_{k=u_{n}}^{v_{n}} A_{n k}\left|Z_{n k}\right|^{r} \\
& =\sum_{k=u_{n}}^{v_{n}} A_{n k}\left(\left|X_{n k}\right|-h^{1 / r}(n)\right)^{r} I\left(\left|X_{n k}\right|^{r}>h(n)\right) \rightarrow 0 \quad \text { a.s. as } n \rightarrow \infty,
\end{aligned}
$$

which implies that $S_{2 n} \rightarrow 0$ a.s. as $n \rightarrow \infty$. 
Now we prove that $S_{1 n} \rightarrow 0$ a.s. as $n \rightarrow \infty$. By the conditional Markov's inequality, we have for all $\varepsilon>0$ that

$$
\begin{aligned}
\sum_{n=1}^{\infty} P^{\mathcal{B}}\left(\left|S_{1 n}\right|>\varepsilon\right) & \leq \frac{1}{\varepsilon} \sum_{n=1}^{\infty} E^{\mathcal{B}}\left|S_{1 n}\right| \\
& \leq \frac{1}{\varepsilon} \sum_{n=1}^{\infty} \sum_{k=u_{n}}^{v_{n}} A_{n k}^{1 / r} E^{\mathcal{B}}\left(\left|Y_{n k}\right|^{r} \cdot\left|Y_{n k}\right|^{1-r}\right) \\
& \leq \frac{1}{\varepsilon} \sum_{n=1}^{\infty} \sum_{k=u_{n}}^{v_{n}} A_{n k}^{1 / r} h^{(1-r) / r}(n) E^{\mathcal{B}}\left|X_{n k}\right|^{r} \\
& \leq \frac{1}{\varepsilon} \sum_{n=1}^{\infty}\left(h(n) \sup _{u_{n} \leq k \leq v_{n}} A_{n k}\right)^{\frac{1-r}{r}} \sum_{k=u_{n}}^{v_{n}} A_{n k} E^{\mathcal{B}}\left|X_{n k}\right|^{r} \\
& \leq \frac{1}{\varepsilon}\left[\sum_{n=1}^{\infty}\left(h(n) \sup _{u_{n} \leq k \leq v_{n}} A_{n k}\right)^{\frac{1-r}{r}}\right] \cdot\left[\sup _{n \geq 1} \sum_{k=u_{n}}^{v_{n}} A_{n k} E^{\mathcal{B}}\left|X_{n k}\right|^{r}\right] \\
& <\infty \text { a.s., } \quad
\end{aligned}
$$

where the third inequality follows from (2.1) and the last inequality follows from condition (ii) and Definition 3.1. Hence, we have by (3.2) and the conditional Borel-Cantelli lemma that

$$
P^{\mathcal{B}}\left(\left|S_{1 n}\right|>\varepsilon, \text { i.o. }\right)=0 \quad \text { a.s. }
$$

which implies that $S_{1 n} \rightarrow 0$ a.s. as $n \rightarrow \infty$ since the $P^{\mathcal{B}}$-null sets and the $P$-null sets coincide (see, e.g., Yuan and Xie, [16]).

Therefore, $S_{n}=S_{1 n}+S_{2 n} \rightarrow 0$ a.s. as $n \rightarrow \infty$. This completes the proof of the theorem.

\section{Competing interests}

The authors declare that they have no competing interests.

\section{Authors' contributions}

All authors read and approved the final manuscript.

\section{Acknowledgements}

The authors are most grateful to the editor and anonymous referee for careful reading of the manuscript and valuable suggestions which helped in improving an earlier version of this paper. This work was supported by the National Natural Science Foundation of China (11201001, 11171001, 11126176, 11226207), the Natural Science Foundation of Anhui Province (1308085QA03, 11040606M12, 1208085QA03), the Specialized Research Fund for the Doctoral Program of Higher Education of China (20093401120001), the 211 project of Anhui University, the Youth Science Research Fund of Anhui University and the Students Science Research Training Program of Anhui University (KYXL2012007).

Received: 5 January 2013 Accepted: 1 March 2013 Published: 22 March 2013

\section{References}

1. Basawa, IV, Prakasa Rao, BLS: Statistical Inference for Stochastic Processes. Academic Press, London (1980)

2. Roussas, GG: On conditional independence, mixing, and association. Stoch. Anal. Appl. 26, 1274-1309 (2008)

3. Leek, JT: Asymptotic conditional singular value decomposition for high-dimensional genomic data. Biometrics 67(2), 344-352 (2011)

4. Ordóñez Cabrera, M, Rosalsky, A, Volodin, A: Some theorems on conditional mean convergence and conditional almost sure convergence for randomly weighted sums of dependent random variables. Test 21, 369-385 (2012)

5. Chow, YS, Teicher, H: Probability Theory: Independence, Interchangeability, Martingales, 3rd edn. Springer, New York (1997) 
6. Prakasa Rao, BLS: Conditional independence, conditional mixing and conditional association. Ann. Inst. Stat. Math 61, 441-460 (2009)

7. Landers, D, Rogge, L: Laws of large numbers for pairwise independent uniformly integrable random variables. Math. Nachr. 130(1), 189-192 (1987)

8. Chandra, TK: Uniform integrability in the Cesàro sense and the weak law of large numbers. Sankhya, Ser. A 51, 309-317 (1989)

9. Ordóñez Cabrera, M: Convergence of weighted sums of random variables and uniform integrability concerning the weights. Collect. Math. 45, 121-132 (1994)

10. Sung, SH: Weak law of large numbers for arrays of random variables. Stat. Probab. Lett. 42, 293-298 (1999)

11. Chandra, TK, Goswami, A: Cesàro $\alpha$-integrability and laws of large numbers I. J. Theor. Probab. 16, 655-669 (2003)

12. Chandra, TK, Goswami, A: Cesàro $\alpha$-integrability and laws of large numbers II. J. Theor. Probab. 19, $789-816$ (2006)

13. Ordóñez Cabrera, M, Volodin, A: Mean convergence theorems and weak laws of large numbers for weighted sums of random variables under a condition of weighted integrability. J. Math. Anal. Appl. 305, 644-658 (2005)

14. Sung, SH, Lisawadi, S, Volodin, A: Weak laws of large numbers for arrays under a condition of uniform integrability. J. Korean Stat. Soc. 45, 289-300 (2008)

15. Yuan, DM, Tao, B: Mean convergence theorems for weighted sums of arrays of residually $h$-integrable random variables concerning the weights under dependence assumptions. Acta Appl. Math. 103, 221-234 (2008)

16. Yuan, DM, Xie, Y: Conditional limit theorems for conditionally linearly negative quadrant dependent random variables. Monatshefte Math. (2012). doi:10.1007/s00605-012-0373-1

doi:10.1186/1029-242X-2013-122

Cite this article as: Shen et al.: Conditional convergence for randomly weighted sums of random variables based on conditional residual $h$-integrability. Journal of Inequalities and Applications 2013 2013:122.

\section{Submit your manuscript to a SpringerOpen ${ }^{\circ}$ journal and benefit from:}

- Convenient online submission

- Rigorous peer review

- Immediate publication on acceptance

- Open access: articles freely available online

- High visibility within the field

- Retaining the copyright to your article 